\title{
Orchidaceae da Reserva Ecológica do Guará, DF, Brasil
}

\author{
João Aguiar Nogueira Batista ${ }^{1,2}$, Luciano de Bem Bianchetti ${ }^{1}$ e Keiko Fueta Pellizzaro ${ }^{1}$
}

Recebido em 21/11/2003. Aceito em 30/08/2004

RESUMO - (Orchidaceae da Reserva Ecológica do Guará, DF, Brasil). É apresentada uma relação de Orchidaceae para a Reserva Ecológica do Guará, pequena unidade de conservação com 194 ha, localizada no Distrito Federal, na região central do Brasil. São registrados 44 gêneros, 100 espécies e cinco táxons subespecíficos. Os grupos mais bem representados são o gênero Habenaria (32 táxons), a subtribo Spiranthinae (seis gêneros e 14 táxons) e o gênero Cyrtopodium (sete espécies). Do total de táxons da Reserva, 80 (76\%) são terrestres e $22(21 \%)$ são epifíticos. Nas fitofisionomias florestais ocorrem 42 táxons, distribuídos segundo um gradiente vertical de umidade e luminosidade. Nas fitofisionomias campestres ocorrem 63 táxons, distribuídos segundo um gradiente horizontal de umidade que vai do campo limpo inundável ao campo sujo seco e cerrado. O campo limpo inundável e o campo limpo estacionalmente úmido, com 42 táxons, são as fitofisionomias com o maior número de táxons. Para todo o bioma cerrado, a Reserva Ecológica do Guará, embora com área pequena, representa a localidade conhecida com maior número de táxons de Orchidaceae. Considerando o conhecimento atual, a Reserva Ecológica do Guará rivaliza ou mesmo sobrepuja, em número de táxons, outras áreas consideradas ricas em diversidade para a família e pertencentes a outros biomas.

Palavras-chave: Orchidaceae, cerrado, Reserva Ecológica do Guará, Distrito Federal, Brasil

\begin{abstract}
Orchidaceae of the Reserva Ecológica do Guará, Distrito Federal, Brazil). A floristic survey of the Orchidaceae of the Reserva Ecológica do Guará, a small conservation unit with 194 ha located in the Distrito Federal, central Brazil, is presented. Forty four genera, 100 species, and five subspecific taxa are recorded for the Reserve. The most representative groups are the genus Habenaria (32 taxa), subtribe Spiranthinae (six genera and 14 taxa) and the genus Cyrtopodium (seven species). Of these, 80 (76\%) are terrestrials, while $22(21 \%)$ are epiphytic. Forty two taxa occur in forest physiognomies, where they are distributed according to vertical gradient of light and humidity. In grassland physiognomies occur 63 taxa, distributed according horizontal gradient of humidity, ranging from the campo limpo inundável (flooded grassland) to the campo sujo seco (dry shrub) and cerrado. The campo limpo inundável and campo úmido (humid grassland), with 42 taxa, are the physiognomies with the highest number of species. The Reserva Ecológica do Guará, though its small area, is the locality in the cerrado biome with the highest number of orchid taxa. Considering the current knowledge, the Reserva Ecológica do Guará rivals or exceeds in number of taxa other orchid-rich areas in other Brazilian biomes.
\end{abstract}

Key words: Orchidaceae, cerrado, Reserva Ecológica do Guará, Distrito Federal, Brazil.

\section{Introdução}

Orchidaceae é, provavelmente, a maior das famílias de angiospermas, compreendendo cerca de 19.500 espécies (Dressler 1993). As orquídeas são de distribuição cosmopolita, mas os principais centros de diversidade são as regiões tropicais da América e Ásia. No continente americano, os principais centros de diversidade são as florestas de altitude, encontradas na faixa média dos Andes, a oeste, e na Serra do Mar, a leste (Pabst \& Dungs 1975). A Colômbia, com cerca de 3.000 espécies, é o país com o maior número registrado de espécies, seguido do Equador e do Brasil com cerca de 2.500 espécies cada (Dressler 1982).
Os naturalistas e exploradores que percorreram o território brasileiro no século passado já destacavam as diferenças em relação à exuberância da vegetação e à grande diversidade de espécies da Mata Atlântica quando comparada aos campos e cerrados no interior do país (Spix \& Martius 1828; Warming 1892). Para a família Orchidaceae essa comparação não é diferente e o cerrado sempre foi considerado como um centro secundário quanto à freqüência e à diversidade dessa família. No Brasil, entre as quatro províncias climáticas estabelecidas para as Orchidaceae por Pabst \& Dungs (1975), o cerrado, com cerca de 500-600 espécies (25\%), aparece na terceira posição em relação ao número total de espécies. Em primeiro lugar está a Serra do Mar, com 1.300-1.400 (60\%) das espécies

\footnotetext{
1 Embrapa Recursos Genéticos e Biotecnologia, prédio da Biotecnologia, C. Postal 02372, CEP 70770-901, Brasília, DF, Brasil

2 Autor para correspondência: janb@ cenargen.embrapa.br
} 
brasileiras, seguida da bacia Amazônica, com cerca de 700-900 espécies (35\%). Entretanto, apesar da aparente importância secundária do bioma cerrado na diversidade geral das Orchidaceae brasileiras, desde o trabalho pioneiro de Warming (1892) até trabalhos mais recentes (Mendonça et al. 1998), Orchidaceae é apontada como uma das cinco famílias mais diversas na flora do cerrado. No mais recente inventário florístico do cerrado, Mendonça et al. (1998) registraram 493 táxons de Orchidaceae.

No cerrado, um dos locais mais bem explorado e pesquisado por botânicos é o Distrito Federal (DF), localizado na região nuclear do bioma, e para onde estão registradas 148 famílias e aproximadamente 3.037 espécies de fanerógamas (Proença et al. 2001). Para a família Orchidaceae, Batista \& Bianchetti (2003) registraram 72 gêneros e 254 táxons. No Distrito Federal há vários trabalhos sobre florística de áreas específicas (Pereira et al. 1993; Maury et al. 1994; Walter \& Sampaio 1998; UNESCO 2000; Nogueira et al. 2002), mas poucos tratam especificamente de Orchidaceae (Batista \& Bianchetti 2003).

O levantamento da flora e das Orchidaceae da Reserva Ecológica do Guará teve inicio durante a década de 1960, com Ezechias P. Heringer, que coletou intensivamente no local o que resultou, para as Orchidaceae, em uma coleção com cerca de 50 espécies. Ainda entre os anos 1960 e 1980, Howard S. Irwin e colaboradores, pesquisadores do Jardim Botânico de Brasília e coletores como Anajulia E. H. Salles coletaram esporadicamente na área da Reserva. Em 1986 iniciou-se um levantamento sistemático das Orchidaceae da Reserva pelos dois primeiros autores do presente trabalho, e que prossegue até a presente data. Durante a década 1990, outros coletores como Zenilton J.G. Miranda, Rafael S. Oliveira, Glocimar Pereira-da-Silva e pesquisadores da Reserva Ecológica do IBGE também coletaram esporadicamente na área da Reserva, resultando em alguns novos registros para o local.

$\mathrm{Na}$ listagem das espécies de fanerógamas do DF, Proença et al. (2001) indicaram sua ocorrência nas principais unidades de conservação, registrando 40 gêneros e 91 táxons de Orchidaceae para a Reserva Ecológica do Guará e o Parque do Guará. Recentemente, Nogueira et al. (2002) apresentaram os resultados de um levantamento florístico do Parque do Guará, uma área vizinha a Reserva Ecológica do Guará, registrando 94 famílias botânicas e 496 espécies. Para Orchidaceae, baseados em versão anterior da lista apresentada no presente trabalho, foram citados
41 gêneros, 94 espécies e quatro variedades, excluindo-se alguns sinônimos e espécies que reconhecidamente não ocorrem no DF.

Este trabalho teve como objetivo apresentar um inventário atualizado das Orchidaceae da Reserva Ecológica do Guará, incluindo dados de coleta, floração, ecologia e conservação das espécies.

\section{Material e métodos}

Localização e caracterização - A Reserva Ecológica do Guará (REG) está localizada entre a cidade satélite do Guará e o Setor de Indústria e Abastecimento (SIA), no DF. A Reserva foi criada em 1988 com o intuito de preservar a mata de galeria na qual se encontra a nascente do Córrego Guará. O córrego Guará pertence à bacia do São Bartolomeu, o qual faz parte da bacia do rio Paraná. Essa unidade de conservação encontra-se dividida em duas partes: uma situada ao norte da Estrada Parque Taguatinga (EPTG) entre os paralelos $15^{\circ} 48^{\prime}-15^{\circ} 50^{\prime}$ 'S e os meridianos $47^{\circ} 57^{\prime}$ 47 59' W, compreendendo 106 ha, e outra, situada ao sul da EPTG, com 41 ha. Posteriormente, a área da Reserva foi aumentada em 47 ha, totalizando 194 ha, mas essa área adicional não foi aqui incluída em função de não haver registros de coleta de Orchidaceae para o local. A área no entorno da reserva encontra-se completamente urbanizada. A sudeste da REG está situado o Parque do Guará, que representa outra unidade de conservação, contígua mas distinta. Anterior à criação da reserva, a área estava subordinada à Fundação Zoobotânica do Distrito Federal (FZDF).

A Reserva do Guará está localizada no pediplano de Brasília, que apresenta altitude média de $1.000 \mathrm{~m}$ e clima do tipo tropical de altitude ( $\mathrm{Cwa}$ ), com temperatura, para o mês mais frio, inferior a $18{ }^{\circ} \mathrm{C}$ e com média superior a $22{ }^{\circ} \mathrm{C}$ no mês mais quente. $\mathrm{O}$ clima da região é marcado por duas estações bem definidas: a estação chuvosa, que vai de outubro a abril e apresenta maior pluviosidade no verão, entre dezembro e março, e concentra mais de $80 \%$ do total anual de chuvas do Distrito Federal, que oscila entre 1.200-1.750 mm; e a estação seca, que se estende de maio a setembro com a umidade relativa do ar, no período, variando de $70 \%$ a menos de $20 \%$ ao final da estação (Codeplan 1984).

Vegetação - Predomina na Reserva, segundo a terminologia proposta por Ribeiro \& Walter (1998), uma mata de galeria inundável, circundanda pelas fitofisionomias campestres, seguindo um gradiente 
horizontal de umidade, de campo limpo inundável, campo limpo estacionalmente úmido (que freqüentemente aparece associado a murundus), campo limpo seco, campo sujo, cerrado ralo e o cerrado propriamente dito. Em relação à composição e distribuição dos táxons de Orchidaceae, a mata de galeria inundável pode ser adicionalmente dividida em duas formas bem distintas. Em um dos tipos, aqui denominado mata de galeria inundável aberta, a cobertura arbórea não é muito desenvolvida, sendo elevada a quantidade de luz que atinge os estratos inferiores. São raras as grandes árvores, predominando arbustos e árvores menores. Nesse ambiente predomina Xylopia emarginata Mart., que domina boa parte da cobertura arbórea. Na outra forma da mata de galeria inundável, aqui denominada mata de galeria inundável fechada, embora o solo seja igualmente inundado, a cobertura arbórea é bem desenvolvida, sendo comum árvores de porte maior, de maneira que o dossel é bem mais fechado e bem menor a quantidade de luz que atinge os estratos inferiores.

Florística - O levantamento das Orchidaceae da Reserva do Guará foi realizado por meio de coletas e do exame de material herborizado depositado nos herbários CEN, HB, HEPH, IBGE e UB. No total foram efetuadas cerca de 70 excursões de coleta, entre 1986 e 2004, com duração média de quatro a seis horas cada. Os "vouchers" destas coletas encontram-se depositados principalmente no herbário CEN. Na seleção do material de outros coletores também foram incluídas coletas provenientes do Setor de Industria (SIA) e, para coletas anteriores a 1988, as localidades Parque do Guará e Horto do Guará, pois até a criação da Reserva, o nome Parque do Guará era aplicado a todas as áreas de preservação ao longo do córrego Guará.

Para a identificação dos táxons foram utilizadas as principais obras de referência na taxonomia das Orchidaceae brasileiras (Cogniaux 1893-1896; 1898-1902; 1904-1906; Hoehne 1940; 1942; 1945; 1953; Pabst \& Dungs 1975; 1977) e alguns trabalhos específicos mais recentes (Garay 1977; 1980; Hágsater 1993; Castro-Neto \& Campacci 2000; Christenson 1988; 1996; Van den Berg \& Chase 2001). No caso de Habenaria e Cyrtopodium as identificações foram feitas principalmente pela comparação com as descrições originais, com os materiais tipos e ilustrações originais, quando disponíveis.

Floração - Os dados de floração foram obtidos tanto das exsicatas quanto de observações feitas em campo pelos autores. Esses dados, em sua maioria, foram obtidos diretamente de material da REG. No entando, em alguns poucos casos, quando os dados não estavam disponíveis, foram considerados dados provenientes de plantas ou populações de outros locais no Distrito Federal. Em outros casos, para algumas das espécies só conhecidas por coletas de E. P. Heringer, com a finalidade de esclarecer tanto o local quanto a época de coleta, os dados das exsicatas foram comparados e corrigidos com o caderno original de coletas do coletor.

\section{Resultados e discussão}

Florística - Foram registrados para a Reserva do Guará 44 gêneros, 100 espécies, quatro variedades e uma forma, totalizando 105 táxons de Orchidaceae (Tab. 1). Dentre os grupos mais representativos destaca-se Habenaria, com 32 táxons (30 spp.). Outros grupos bem representados na Reserva são a subtribo Spiranthinae, com seis gêneros e 14 táxons (13 spp.) e Cyrtopodium (7 spp.). Destaca-se, também, a ocorrência de algumas espécies raras ou pouco coletadas no âmbito regional, ou mesmo no Cerrado, como Cyanaeorchis minor, Cycnoches pentadactylum, Govenia utriculata, Habenaria achalensis, $H$. pungens, $H$. regnellii, Phragmipedium vittatum, Sarcoglottis homalogastra e S. sagittata.

Do total de 105 táxons registrados para a área, 80 (76\%) são terrestres, 22 (21\%) são epífitas, dois (2\%), representados por Vanilla, são escandentes e um (1\%), Epidendrum secundum, cresce tanto como terrestre como epifítico (Tab. 1). Das terrestres, 63 (79\%) ocorrem em formações campestres e 17 (21\%) crescem no interior da mata de galeria. Todas as espécies epífitas ocorrem na mata de galeria e uma única, Bulbophyllum insectiferum, também pode ser encontrada sobre buritis (Mauritia flexuosa Mart.) no campo inundável. A predominância de terrestres sobre epífitas contrasta notadamente com a forma de vida das Orchidaceae na Mata Atlântica onde se observa a relação inversa.

Até o momento foram descritos cinco táxons a partir de material proveniente da REG (Pabst 1967; 1976; Menezes 1995a; 1995b): Habenaria heringeri, Rodriguezia decora var. lactea, Cyrtopodium fowliei, C. paludicolum var. reginae e Oncidium hydrophilum var. immaculatum, sendo os dois últimos sinônimos de $C$. paludicolum e $O$. hydrophilum, respectivamente. Dos táxons registrados para a REG, três $(2,9 \%)$ parecem ser restritos à Reserva, Rodriguezia decora var. lactea, Habenaria aff. 
aphylla 1 e $H$. aff. aphylla 2 , sendo os dois últimos, possivelmente, táxons infraespecíficos de $H$. aphylla, ainda não descritos. Também foi observado na REG um exemplar de Galeandra paraguayensis com flores sem pigmentos e um exemplar de Epidendrum secundum, com flores completamente brancas, mas nenhuma destas "formas" se encontra descrita. Outra espécie, Cycnoches pentadactylum, embora de distribuição bastante ampla, é conhecida no Distrito Federal apenas por uma coleta proveniente da REG. Liparis nervosa f. kappleri é conhecida para o Brasil, apenas da REG e, até onde foi possível averiguar (Christenson 1996), corresponde à segunda coleta desse táxon. Portanto, do total de 254 táxons de Orchidaceae registrados para o Distrito Federal (Batista \& Bianchetti 2003), cinco (2\%) são conhecidos apenas para a Reserva.

Dos 105 táxons de Orchidaceae registrados para a Reserva 16 (15\%) são indicados para o local somente pelas coletas de E.P. Heringer ou H.S. Irwin (Tab. 1) e não foram mais coletados ou observados no local nos últimos 37 anos. Várias explicações poderiam ser formuladas para esclarecer porque estas espécies não foram mais encontradas. Uma possibilidade é que elas tenham sido coletadas em outros locais ao longo do córrego Guará, fora da área atual da Reserva. Entretanto, embora tenha-se percorrido alguns pontos ao longo do córrego Guará, abaixo da Reserva, fora de sua área, a região encontra-se muito alterada e pouco resta da vegetação nativa original. Outra possibilidade é que estes táxons apresentem naturalmente uma freqüência muito baixa na área da Reserva. De acordo com esta possibilidade, Warming (1892) relatou uma frequiência baixíssima para algumas Orchidaceae na região de Lagoa Santa, só tendo encontrado algumas espécies uma única ocasião nos três anos que passou no local. Este pode ser o caso do Cycnoches pentadactylum e Govenia utriculata, esta última também muito pouco comum no Distrito Federal. Considerando as terrestres, a sazonalidade poderia explicar a situação de algumas espécies: uma grande população, fora de uma determinada época, pode ocorrer em determinado local sem que haja condições favoráveis para o crescimento e floração das plantas, passando assim desapercebida, como acontece com diversas espécies de Habenaria. Outra possibilidade é que algumas destas espécies ocorram em locais da Reserva pouco explorados. Esse pode ser o caso de Epidendrum dendrobioides e Habenaria edwallii, que são espécies de ambiente muito úmido e de difícil acesso. Por fim, não se descarta a possibilidade de erro ou engano na localização exata do ponto de coleta das plantas. Na década de 1960 a Fundação Zoobotânica era responsável por vários locais que hoje constituem áreas de preservação, como a REG e a Área de Relevante Interesse Ecológico (ARIE) do Riacho Fundo (localizada no final do córrego Guará, quando este desemboca no córrego Riacho Fundo), e, naquela época, nem sempre os coletores discriminavam muito bem estes locais.

Quatro espécies incluídas na lista (Tab. 1) não apresentam um exemplar testemunha correspondente. A inclusão delas para a Reserva é baseada na observação de plantas em floração encontradas no local pelos autores (Cyrtopodium brandonianum, Habenaria alpestris e Lanium avicula), ou por Zenilton J.G. Miranda (Isochillus linearis) e que não foram herborizadas.

Nos inventários florísticos apresentados para a REG e Parque do Guará (Proença et al. 2001; Nogueira et al. 2002), Orchidaceae aparece como a família com o maior número de espécies, chegando a representar 20\% do total de espécies registradas para o local. Este valor é maior do que o observado para outras localidades, tanto dentro como fora do DF. Entre alguns exemplos, para a R.E. do IBGE esta proporção é de 4\% (Pereira et al. 1993), para Lagoa Santa 4,6\% (Warming 1892), para a Serra do Cipó 5\% (Barros 1987), para Pico das Almas (Toscano-de-Brito 1995) e Catolés cerca de 4,4\% (Zappi et al. 2003), para a Ilha do Cardoso chega a 12\% (Barros 1991), e para o DF 7,1\% (Proença et al. 2001). Pelo esforço de coleta específico voltado para Orchidaceae, a amostragem dessa família para a REG é certamente maior do que para outras famílias botânicas, podendo assim superestimar este valor. De qualquer modo, esses dados evidenciam a riqueza incomum da Reserva em representantes da família.

Por fim, a listagem de Orchidaceae apresentada por Nogueira et al. (2002) para o Parque do Guará deve ser revisada, pois é, fundamentalmente, baseada numa versão anterior da lista apresentada no presente trabalho (Tab. 1) e esta não inclui coletas realizadas no atual Parque do Guará.

Distribuição por fitofisionomia - Na mata de galeria inundável aberta ocorrem 20 táxons de Orchidaceae (Tab. 2), distribuídos em função de gradientes verticais de luminosidade e umidade. No chão inundado, mas sempre sobre bolsões de terra firme, com água circundante, ocorrem 10 táxons. Aparecem em quantidade algumas Spiranthinae como Pelexia pterygantha, P. hypnophila e Sauroglossum 
Tabela 1. Lista das espécies de Orchidaceae da Reserva Ecológica do Guará, com sua respectiva forma de vida, número de coletas, número de registros, época de floração, hábitat e material-testemunho e respectivo herbário. Forma de vida: T, terrestre; E, epifítico; S, escandente. Habitat: VER, vereda; MG, mata de galeria; MGF, mata de galeria inundável fechada; MGA, mata de galeria inundável aberta; CI, campo limpo inundável; CEU, campo limpo estacionalmente úmido; CS, campo limpo seco; CSJ, campo sujo; CER, cerrado ralo e cerrado.

Táxons Forma № coletas/ Floração Hábitat Material-testemunho
de vida registros $^{\mathrm{b}}$

Bletia catenulata Ruiz \& Pav.

Brassavola cf. rhomboglossa Pabst

Bulbophyllum insectiferum Barb. Rodr.

Cattleya bicolor Lindl.

Campylocentrum neglectum (Rchb. f. \& Warm.) Cogn.

Cleistes bella Rchb.f. \& Warm.

C. caloptera Rchb.f. \& Warm.

C. castanoides Hoehne

C. paranaensis (Barb. Rodr.) Schltr.

Cleistes cf. tenuis Rchb. f.

Comparettia coccinea Lindl.

Cranichis glabricaulis Hoehne

Cyanaeorchis minor Schltr.

Cycnoches pentadactylum Lindl. ${ }^{\mathrm{a}}$

Cyrtopodium brandonianum Barb. Rodr.

C. brunneum J.A.N. Bat. \& Bianchetti

C. fowliei L.C. Menezes

C. pallidum Rchb. f. \& Warm.

C. paludicolum Hoehne

C. parviflorum Lindl.

C. poecilum Rchb. f. \& Warm.

Encyclia osmantha (Barb. Rodr.) Schltr. ${ }^{a}$

Epidendrum dendrobioides Thumb. ${ }^{\mathrm{a}}$

E. densiflorum Lindl.

E. difforme Jacq.

E. nocturnum Jacq.

E. secundum Jacq.

Epistephium lucidum Cogn.

E. sclerophyllum Lindl.

Erythrodes arietina (Rchb. f. \& Warm.) Ames

Galeandra beyrichii Rchb. f. ${ }^{\mathrm{a}}$

G. montana Barb. Rodr.

G. paraguayensis Cogn.

G. styllomisantha (Vell.) Hoehne

Galeottia ciliata (Morel) Dressler \& Christenson

Govenia utriculata (Sw.) Lindl. ${ }^{\mathrm{a}}$

Habenaria achalensis Kraenzl. ${ }^{\mathrm{a}}$

H. alpestris Cogn.

H. anisitsii Kraenzl. ${ }^{\mathrm{a}}$

Habenaria aff. aphylla Barb. Rodr. (1)

Habenaria aff. aphylla Barb. Rodr. (2)

$H$. ayangannensis Renz.

H. balansaei Cogn.

H. brevidens Lindl. ${ }^{\mathrm{a}}$

Habenaria aff. coxipoensis Hoehne ${ }^{\mathrm{a}}$

$H$. curtbradei Hoehne

H. curvilabria Barb.Rodr. ${ }^{\mathrm{a}}$

H. edwallii Cogn. ${ }^{\mathrm{a}}$

Habenaria aff. edwallii Cogn.

H. glaucophylla Barb. Rodr. var. brevifolia Cogn.

H. gourlieana Gill. ex Lindl.

H. graciliscapa Barb. Rodr.

\begin{tabular}{|c|c|c|c|c|}
\hline $\mathrm{T}$ & $3 / 20$ & Jul-Dez & CI, CEU & Batista 85 - CEN \\
\hline $\mathrm{E}$ & $3 / 9$ & Set-Out & MGA, MGF & Batista $119-$ CEN \\
\hline $\mathrm{E}$ & $1 / 3$ & Ago & MGF, VER & Heringer 8447 - HB \\
\hline $\mathrm{E}$ & $3 / 13$ & Jan-Fev & MGA & Oliveira 72 - UB \\
\hline $\mathrm{E}$ & $1 / 1$ & Out & MG & Batista 934 - CEN \\
\hline $\mathrm{T}$ & $1 / 4$ & Jan-Fev & CS, CSJ & Heringer 9690 - UB \\
\hline $\mathrm{T}$ & $1 / 1$ & Jan-Fev & CEU & Oliveira 76 - UB \\
\hline $\mathrm{T}$ & $8 / 15$ & Nov-Dez & CEU & Bianchetti \& Batista 819 - CEN \\
\hline $\mathrm{T}$ & $1 / 2$ & Dez-Jan & CS & Heringer 9680 - UB \\
\hline $\mathrm{T}$ & $1 / 2$ & Nov & CEU & Miranda 23 - CEN \\
\hline $\mathrm{E}$ & $3 / 5$ & Fev-Mar & MGF & Oliveira 16 - UB \\
\hline $\mathrm{T}$ & $2 / 2$ & Mai & MG & Heringer 8323 - HB \\
\hline $\mathrm{T}$ & $5 / 5$ & Out & CI, CEU & Batista 130 - CEN \\
\hline $\mathrm{E}$ & $1 / 1$ & Mar & MG & Heringer 8033 - UB \\
\hline $\mathrm{T}$ & $0 / 2$ & Jan & $\mathrm{CS}, \mathrm{CSJ}$ & sem voucher \\
\hline $\mathrm{T}$ & $4 / 7$ & Set & CSJ, CER & Batista 330 - CEN \\
\hline $\mathrm{T}$ & $10 / 21$ & Out-Dez & CEU & Batista 1269 - CEN (Epitypus) \\
\hline $\mathrm{T}$ & $3 / 6$ & Nov-Dez & $\mathrm{CS}$ & Batista 132 - CEN \\
\hline $\mathrm{T}$ & $6 / 15$ & Dez-Mai & CI & Bianchetti \& Batista 840 - CEN \\
\hline $\mathrm{T}$ & $3 / 14$ & Ago-Set & CEU & Bianchetti \& Batista 951 - CEN \\
\hline $\mathrm{T}$ & $1 / 2$ & Out-Nov & $\mathrm{CS}$ & Batista 114 - CEN \\
\hline $\mathrm{E}$ & $1 / 1$ & Jul & MG & Heringer 8453-647 - UB \\
\hline $\mathrm{T}$ & $3 / 3$ & Dez-Fev & $\mathrm{CI}$ & Heringer 8520 - UB \\
\hline $\mathrm{E}$ & $3 / 14$ & Ago-Set & MGF & Batista 33 - HEPH \\
\hline $\mathrm{E}$ & $3 / 7$ & Dez-Fev & MGA, MGF & Bianchetti \& Batista 839 - CEN \\
\hline $\mathrm{E}$ & $3 / 12$ & Nov-Abr & MGA & Bianchetti \& Batista 838 - CEN \\
\hline $\mathrm{T} / \mathrm{E}$ & $2 / 5$ & Out-Fev & MGA & Heringer 8774-966 - UB \\
\hline $\mathrm{T}$ & $2 / 6$ & Fev-Mar & MGA & Heringer 8064 - UB \\
\hline $\mathrm{T}$ & $4 / 4$ & Dez-Abr & CS, CSJ, CER & Heringer 7915 - UB \\
\hline $\mathrm{T}$ & $4 / 12$ & Jun-Set & MGF & Batista 86 - CEN \\
\hline $\mathrm{T}$ & $2 / 2$ & Fev & MG & Heringer 7991 - UB \\
\hline $\mathrm{T}$ & $2 / 3$ & Mar & CS, CSJ, CER & Batista 64A - CEN \\
\hline $\mathrm{T}$ & $5 / 12$ & Out-Nov & CEU & Batista 423 - CEN \\
\hline $\mathrm{T}$ & $1 / 1$ & Dez-Jan & CEU & E.J.B.B. 1068 - HEPH \\
\hline $\mathrm{E}$ & $4 / 15$ & Out-Jan & MGA & Batista 38 - CEN \\
\hline $\mathrm{T}$ & $1 / 1$ & Fev & MGF & Heringer 7996 - UB \\
\hline $\mathrm{T}$ & $1 / 1$ & Dez & CI & Heringer 10815 - HB \\
\hline $\mathrm{T}$ & $0 / 1$ & Jan & CEU & sem voucher \\
\hline $\mathrm{T}$ & $3 / 3$ & Dez & CI & Heringer 9984 - HB \\
\hline $\mathrm{T}$ & $3 / 6$ & Nov-Dez & CEU & Batista 636 - CEN \\
\hline $\mathrm{T}$ & $3 / 7$ & Dez-Jan & $\mathrm{CI}$ & Batista 136 - CEN \\
\hline $\mathrm{T}$ & $2 / 2$ & Mar & CEU & Batista 066 - CEN \\
\hline $\mathrm{T}$ & $4 / 4$ & Out-Nov & CI & Batista 744 - CEN \\
\hline $\mathrm{T}$ & $1 / 1$ & Dez & $\mathrm{CS}, \mathrm{CSJ}$ & Heringer 10837 - HB, UB \\
\hline $\mathrm{T}$ & $1 / 1$ & Mar & CSJ, CER & Irwin et al. 14106 - UB \\
\hline $\mathrm{T}$ & $7 / 8$ & Dez-Jan & CS & Batista 1097 - CEN \\
\hline $\mathrm{T}$ & $1 / 1$ & Fev & MG & Heringer 11036 - HB \\
\hline $\mathrm{T}$ & $1 / 1$ & Nov & $\mathrm{CI}$ & Heringer 9213 - HB, UB \\
\hline $\mathrm{T}$ & $1 / 1$ & Jan & CI & $\begin{array}{l}\text { Aparecida-Silva \& Alvarenga } \\
4249 \text { - IBGE }\end{array}$ \\
\hline $\mathrm{T}$ & $6 / 9$ & Mar-Jun & MGA & Batista 64 - CEN \\
\hline $\mathrm{T}$ & $5 / 9$ & Out-Nov & CI & Batista 344 - CEN \\
\hline $\mathrm{T}$ & $9 / 10$ & Dez-Mar & CEU & Batista 39 - CEN \\
\hline
\end{tabular}


Tab. 1 (continuação)

Táxons $\quad \begin{array}{rlll}\begin{array}{c}\text { Forma } \\ \text { de vida }\end{array} & \begin{array}{c}\text { N. coletas/ Floração } \\ \text { registros }\end{array}\end{array}$ Hábitat Material-testemunho

H. guilleminii Rchb. f. ${ }^{\mathrm{a}}$

H. heringeri Pabst

H. juruenensis Hoehne

H. leucosantha Barb.Rodr.

H. longipedicellata Hoehne

Habenaria aff. longipedicellata Hoehne

H. mitomorpha Krzl.

Habenaria aff. mystacina Lindl.

H. nuda Lindl. var. pygmaea Hoehne

H. obtusa Lindl.

H. petalodes Lindl. ${ }^{a}$

H. pungens Cogn.

H. regnellii Cogn.

H. secundiflora Barb.Rodr.

Habenaria aff. sprucei Cogn.

H. trifida Kunth

Houlletia juruensis Hoehne

Isabelia violacea (Lindl.) Van den Berg \& M.W.Chase

Isochillus linearis (Jacq.) R.Br.

Ionopsis utricularioides (Sw.) Lindl.

Lanium avicula (Lindl.) Benth.

Liparis bifolia Cogn.

L. nervosa (Thunb.) Lindl.

L. nervosa f. kappleri (Rchb.f.) Christenson \& Carnevali $\mathrm{T}$

Lyroglossa grisebachii (Cogn.) Schltr.

Malaxis sp.

Mormodes sinuata Rchb.f. \& Warm.

Oeceoclades maculata (Lindl.) Lindl.

Oncidium hydrophilum Barb.Rodr.

Pelexia cuculligera (Rchb.f. \& Warm.) Schltr.

P. goyazensis (Cogn.) Garay

P. hypnophila (Barb.Rodr.) Schltr.

P. oestrifera (Rchb.f. \& Warm.) Schltr. ${ }^{a}$

P. pterygantha (Rchb.f. \& Warm.) Schltr.

Phragmipedium vittatum (Vell.) Rolfe

Platythelys paranaensis (Kraenzl.) Garay

Pleurothallis ramosa Barb.Rodr.

Polystachya sp.

Prescottia microrhiza Barb.Rodr.

Pteroglossa macrantha (Rchb.f.) Schltr.

Rodriguezia decora (Lem.) Rchb.f.

$R$. decora var. lactea L.C. Menezes

Sacoila lanceolata (Aubl.) Garay aff. var. paludicola Luer

Sanderella discolor (Barb.Rodr.) Cogn.

Sarcoglottis biflora (Vell.) Schltr.

S. homalogastra (Rchb.f. \& Warm.) Schltr.

S. sagittata (Rchb.f. \& Warm.) Schltr.

S. simplex (Griseb.) Cogn.

S. uliginosa Barb.Rodr.

Sauroglossum nitidum (Vell.) Schltr.

Scaphyglottis cuneata Schltr. ${ }^{\mathrm{a}}$

Vanilla bahiana Hoehne

V. edwallii Hoehne

\begin{tabular}{|c|c|c|c|c|}
\hline $\mathrm{T}$ & $1 / 1$ & Mar & CEU & Heringer 8122 - HB, UB \\
\hline $\mathrm{T}$ & $8 / 14$ & Dez-Jan & CEU, CS & Heringer 7833 - HB (Holotypus) \\
\hline $\mathrm{T}$ & $8 / 10$ & Dez-Mar & CEU, CS & Batista 55 - CEN \\
\hline $\mathrm{T}$ & $5 / 13$ & Out-Dez & $\mathrm{CI}$ & Batista \& Oliveira-Neto 1088 - CEN \\
\hline $\mathrm{T}$ & $7 / 7$ & Dez-Fev & $\mathrm{CS}$ & Batista 137 - CEN \\
\hline $\mathrm{T}$ & $8 / 11$ & Out-Dez & CEU & Batista 637 - CEN \\
\hline $\mathrm{T}$ & $2 / 2$ & Mar & CEU & Batista 65 - CEN \\
\hline $\mathrm{T}$ & $20 / 29$ & Out-Fev & $\mathrm{CI}, \mathrm{CEU}$ & Bianchetti \& Batista 826 - CEN \\
\hline $\mathrm{T}$ & $12 / 18$ & Jun-Ago & $\mathrm{CI}$ & Batista 87 - CEN \\
\hline $\mathrm{T}$ & $7 / 8$ & Dez-Jan & $\mathrm{CS}, \mathrm{CSJ}$ & Heringer 8128 - HB \\
\hline $\mathrm{T}$ & $1 / 1$ & Mai & MG & Heringer 8320 - UB, HB \\
\hline $\mathrm{T}$ & $2 / 2$ & Jun & CEU & Batista 1 - HEPH \\
\hline $\mathrm{T}$ & $1 / 1$ & Nov & CI & Oliveira 77 - UB \\
\hline $\mathrm{T}$ & $6 / 8$ & Dez-Fev & $\mathrm{CEU}, \mathrm{CS}$ & Bianchetti \& Batista 835 - CEN \\
\hline $\mathrm{T}$ & $1 / 1$ & Nov & CEU & Batista 16 - CEN \\
\hline $\mathrm{T}$ & $3 / 3$ & Dez-Jan & $\mathrm{CS}$ & Batista 138 - CEN \\
\hline $\mathrm{T}$ & $1 / 7$ & Nov & MGA & Bianchetti \& Batista 837 - CEN \\
\hline $\mathrm{E}$ & $2 / 7$ & Ago & MGF & Batista 937 - CEN \\
\hline $\mathrm{E}$ & $0 / 1$ & Jul & MGF & sem voucher \\
\hline $\mathrm{E}$ & $1 / 2$ & Ago-Set & MGA & Batista 935 - CEN \\
\hline $\mathrm{E}$ & $0 / 4$ & Jan-fev & MGF & sem voucher \\
\hline $\mathrm{T}$ & $1 / 1$ & Jan-Fev & CEU & Miranda 6 - UB \\
\hline $\mathrm{T}$ & $1 / 5$ & Dez-Jan & MGA & Bianchetti 844 - CEN \\
\hline $\mathrm{T}$ & $1 / 1$ & Jan & MGA & Batista 62A - CEN \\
\hline $\mathrm{T}$ & $5 / 10$ & Nov-Dez & CEU & Bianchetti \& Batista 828 - CEN \\
\hline $\mathrm{T}$ & $1 / 1$ & Fev & MG & Salles 56 - IBGE \\
\hline $\mathrm{E}$ & $1 / 1$ & Abr & MGF & Batista 197 - CEN \\
\hline $\mathrm{T}$ & $1 / 5$ & Dez-Abr & MGA, MGF & Batista 938 - CEN \\
\hline $\mathrm{T}$ & $3 / 8$ & Out-Dez & CI, CEU & Batista 118 - CEN \\
\hline $\mathrm{T}$ & $4 / 5$ & Out-Nov & $\mathrm{CS}$ & Batista 212 - CEN \\
\hline $\mathrm{T}$ & $1 / 2$ & Set & $\mathrm{CS}$ & Batista 329 - CEN \\
\hline $\mathrm{T}$ & $4 / 15$ & Ago-Set & MGA & Batista 331 - CEN \\
\hline $\mathrm{T}$ & $1 / 1$ & Nov-Dez & $\mathrm{CS}$ & Heringer 9695 - UB \\
\hline $\mathrm{T}$ & $4 / 15$ & Jul-Ago & MGA & Batista 88 - CEN \\
\hline $\mathrm{T}$ & $1 / 17$ & Dez-Abr & $\mathrm{CI}$ & Lima 42 - HEPH \\
\hline $\mathrm{T}$ & $2 / 2$ & Dez-Jan & CEU & Bianchetti \& Batista 845 - CEN \\
\hline $\mathrm{E}$ & $2 / 9$ & Dez-Jan & MGA, MGF & Salles 1525 - HEPH \\
\hline $\mathrm{E}$ & $1 / 3$ & Dez-Jan & MGF & Batista et al. 1453 - CEN \\
\hline $\mathrm{T}$ & $1 / 1$ & Ago & MGA & Batista \& Bianchetti 1241 - CEN \\
\hline $\mathrm{T}$ & $2 / 2$ & Out & $\mathrm{CS}$ & Batista 116 - CEN \\
\hline $\mathrm{E}$ & $2 / 9$ & Jan-Abr & MGA & Bianchetti et al. 853 - CEN \\
\hline $\mathrm{E}$ & $1 / 1$ & Fev & MGA & Menezes 36 - UB (Holotypus) \\
\hline $\mathrm{T}$ & $3 / 8$ & Set-Out & CI & Batista 327 - CEN \\
\hline $\mathrm{E}$ & $1 / 1$ & Mai & MGF & Batista et al. 1454 - CEN \\
\hline $\mathrm{T}$ & $4 / 5$ & Out & CS & Batista 129 - CEN \\
\hline $\mathrm{T}$ & $1 / 2$ & Set & $\mathrm{CS}$ & Batista et al. 1451 - CEN \\
\hline $\mathrm{T}$ & $1 / 1$ & Set & $\mathrm{CS}$ & Batista 328 - CEN \\
\hline $\mathrm{T}$ & $2 / 4$ & Nov-Dez & $\mathrm{CI}$ & Batista 09 - CEN \\
\hline $\mathrm{T}$ & $5 / 16$ & Set-Dez & $\mathrm{CI}$ & Bianchetti \& Batista 827 - CEN \\
\hline $\mathrm{T}$ & $5 / 18$ & Jun-Ago & MGA, MGF & Batista 89 - CEN \\
\hline $\mathrm{E}$ & $1 / 1$ & Mar & MG & Heringer 8055 - UB \\
\hline $\mathrm{S}$ & $2 / 5$ & Set-Out & MGF & Batista 940 - CEN \\
\hline $\mathrm{S}$ & $1 / 3$ & Dez & MGF & Batista 941 - CEN \\
\hline
\end{tabular}

a - Táxons conhecidos somente de coletas de E.P. Heringer ou H.S. Irwin e não coletados ou observados na Reserva nos últimos 37 anos. b Os registros incluem todas as coletas documentadas para a reserva adicionado de observações e registros feitos em campo pelos autores. Abreviações. 
Tabela 2. Número de táxons de Orchidaceae da Reserva Ecológica do Guará por fitofisionomia*.

\begin{tabular}{lc}
\hline Fitofisionomia & № táxons \\
\hline Mata de galeria & 9 \\
Mata de galeria inundável fechada & 18 \\
Mata de galeria inundável aberta & 20 \\
Campo limpo inundável & 20 \\
Campo limpo estacionalmente úmido & 26 \\
Campo limpo seco & 22 \\
Campo sujo & 8 \\
Cerrado e cerrado ralo & 4 \\
\hline
\end{tabular}

* Definidas segundo Ribeiro \& Walter (1998).

nitidum, além de Habenaria glaucophylla var. brevifolia, Houlletia juruensis, Liparis nervosa, Epistephium lucidum, Oeceoclades maculata e, menos frequentemente, Prescottia microrhiza e Liparis nervosa f. kappleri. Entre as epífitas, na parte inferior das árvores e arbustos, mas com as raízes atingindo normalmente o solo, aparecem em quantidade Galleotia ciliata e Epidendrum nocturnum. Em nível superior, na parte mediana das árvores e arbustos, em locais mais secos e expostos a maior insolação, aparece em profusão Rodriguezia decora e, em menor quantidade, Brassavola cf. rhomboglossa, Cattleya bicolor, Epidendrum difforme, Ionopsis utricularioides e Pleurothallis ramosa. Epidendrum secundum, ocorre nas bordas da mata, crescendo preferencialmente como epífita e ocasionalmente como terrestre.

$\mathrm{Na}$ mata de galeria inundável fechada, as Orchidaceae terrestres são menos comuns tanto em número como em freqüência, somente sendo encontradas quatro espécies: Erythrodes arietina, Govenia utriculata, Oeceoclades maculata e Sauroglossum nitidum. Nesse ambiente predominam as epifitas que se restringem à copa das árvores, sendo representadas por Epidendrum difforme, Lanium avicula e Isabelia violacea, Brassavola cf. rhomboglossa, Comparettia coccinea, Polystachya sp., Bulbophyllum insectiferum, Isochillus linearis, Mormodes sinuata, Sanderella discolor e Campylocentrum neglectum. Algumas exceções são Epidendrum densiflorum e Pleurothallis ramosa, comuns nesse ambiente, mas que ocorrem preferencialmente na parte mais baixa da vegetação, principalmente nos pontos mais centrais da mata onde a umidade é maior. Ocorrem também nesta fisionomia Vanilla bahiana e V. edwallii. Cinco espécies, Brassavola cf. rhomboglossa, Epidendrum difforme,
Oeceoclades maculata, Pleurothallis ramosa e Sauroglossum nitidum ocorrem nas duas formas de mata de galeria inundável. Outras oito espécies que ocorrem em matas, Cranichis glabricaulis, Cycnoches pentadactylum, Encyclia osmantha, Galeandra beyrichii, Habenaria curvilabria, $H$. petalodes, Malaxis sp. e Scaphyglottis cuneata, são conhecidas apenas de exsicatas coletada por E.P. Heringer ou A.E.H. Salles e o seu ambiente exato de ocorrência na Reserva é desconhecido.

O número de táxons de Orchidaceae encontrados nas duas formas de mata de galeria inundável é aproximadamente o mesmo, mas impressionam, na forma aberta, a quantidade de indivíduos e a facilidade de observação das plantas, enquanto na forma fechada as plantas aparecem em menor quantidade e, freqüentemente, concentram-se nas copas das árvores, o que torna a sua observação mais difícil.

Nas fitofisionomias campestres ocorrem 63 táxons de Orchidaceae e observa-se um gradiente horizontal de distribuição em função do tipo de solo e do seu grau de umidade. No campo limpo inundável ocorrem 20 táxons (Tab. 2), sendo típicas do local Cyrtopodium paludicolum, Phragmipedium vittatum e, no limite entre a mata e o campo, Habenaria gourlieana. Em anos durante os quais a área é atingida pelo fogo, podem aparecer em profusão Cyanaeorchis minor, Habenaria leucosantha e Sarcoglottis uliginosa. Bletia catenulata também é comum nesse tipo de ambiente, mas juntamente com Habenaria aff. mystacina, Oncidium hydrophilum e Sarcoglottis simplex, também ocorre em campo limpo estacionalmente úmido.

O campo limpo estacionalmente úmido, com 26 táxons, representa a fitofisionomia com o maior número de espécies na Reserva (Tab. 2). Aparecem no local Cyrtopodium fowliei, C. parviflorum, Cleistes castanoides, Galeandra paraguayensis, Lyroglossa grisebachii, Platythelys paranaensis e várias Habenaria. No campo limpo seco podem ser encontrados 22 táxons de Orchidaceae (Tab. 2). São espécies típicas do local Cyrtopodium pallidum, que em anos de queimada pode aparecer em grande quantidade, os raros Pelexia goyazensis e Sarcoglottis sagittata, Pteroglossa macrantha e algumas Habenaria como, $H$. trifida e $H$. longipedicellata. No seu limite superior o campo limpo seco é substituído pelo campo sujo, cerrado ralo e cerrado típico, sendo estas últimas as fitofisionomias com o menor número de espécies de Orchidaceae na Reserva (Tab. 2). As espécies mais típicas e mais 
facilmente observadas nesse ambiente são Habenaria obtusa e Epistephium sclerophyllum.

Períodos de floração - O fator ambiental mais evidentemente relacionado à floração das espécies de Orchidaceae na Reserva é a frequiência e intensidade das chuvas ao longo dos meses do ano, uma vez que, dos 105 táxons registrados para a área, 84 (80\%) florescem durante o período chuvoso, de outubro a abril (Fig. 1). Tomando como ponto de partida o periodo que corresponde ao extremo final do período chuvoso e inicio da estação seca (abril a maio), esta é a época com o menor número de espécies em floração. Nas fisionomias campestres quase não se observa nenhuma Orchidaceae em floração, à exceção de alguns exemplares de Cyrtopodium paludicolum. No interior da mata podem ser encontrados alguns exemplares, em final de floração, de Habenaria glaucophylla var. brevifolia. Entre junho e julho floresce nos campos úmidos Habenaria pungens e começam a aparecer os primeiros exemplares de $H$. nuda var. pygmaea, cuja floração avança até o auge do período seco, entre julho e agosto. No interior da mata inundável começam a florescer os primeiros exemplares de Sauroglossum nitidum e Erythrodes arietina. O auge do período seco é marcado pela floração das Spiranthinae no interior da mata de galeria inundável aberta, principalmente Pelexia pterygantha e $P$. hypnophila, que aparecem em profusão. Entre as epífitas, florescem, neste período, Bulbophyllum insectiferum, Epidendrum densiflorum, Ionopsis utricularioides e Isabelia violacea. Nas fisionomias campestres a floração ainda é incipiente; floresce Cyrtopodium parviflorum e os primeiros exemplares de Bletia catenulata.

No final da seca e começo das chuvas, entre setembro e outubro, começam a florescer as terrestres mais precoces nas fisionomias campestres. Em áreas mais secas florescem Cyrtopodium brunneum, Pteroglossa macrantha, Sarcoglottis biflora e as raras Pelexia goyazensis, Sarcoglottis homalogastra e $S$. sagitatta. Em áreas mais úmidas florescem Bletia catenulata, Sarcoglottis uliginosa, Sacoila lanceolata aff. var. paludicola, Cyrtopodium fowliei e Cyanaeorchis minor, que em alguns anos podem aparecer em grande quantidade. Florescem também as espécies mais precoces de Habenaria como $H$. gourlieana, $H$. balansae e $H$. leucosantha. Na mata florescem tipicamente nesta época Brassavola cf. rhomboglossa, Campylocentrum neglectum e Vanilla bahiana, e começam a aparecer os primeiros exemplares floridos de Epidendrum secundum e Galeottia ciliata.

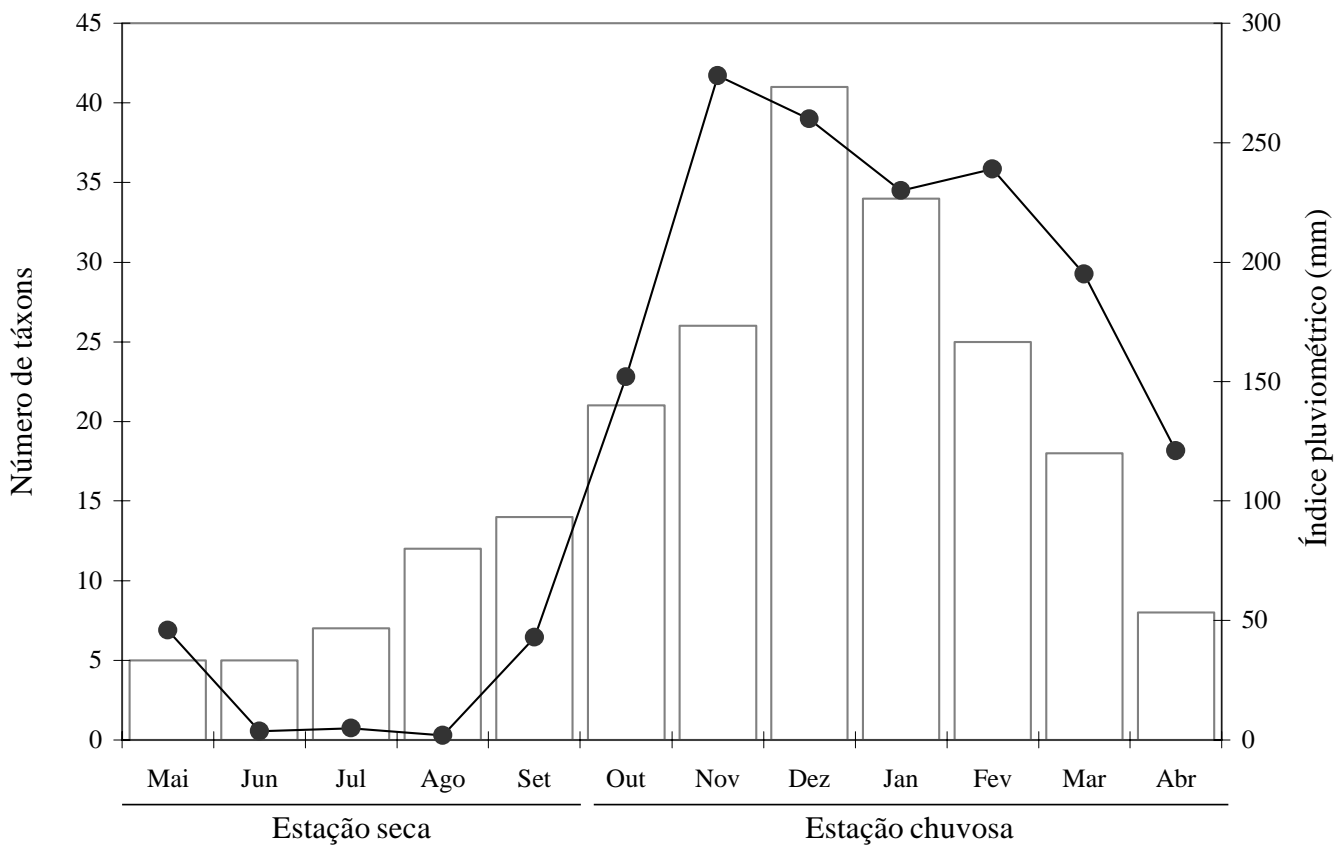

Figura 1. Número de táxons de Orchidaceae floridos por mês na Reserva Ecológica do Guará (REG). O índice pluviométrico está indicado por uma linha. A figura inclui dados de floração de todas as coletas de Orchidaceae registradas para a REG e de observações feitas em campo pelos autores. 
À medida em que as chuvas vão se tornando mais freqüentes, a partir de outubro, florescem nos campos Galeandra paraguayensis, Cyrtopodium poecilum, Oncidium hydrophilum e Pelexia cuculligera e, no interior da mata, Houlletia juruensis. Entre novembro e janeiro as chuvas se tornam ainda mais freqüentes e intensas, marcando o auge do período chuvoso. Floresce nos campos a grande maioria das espécies de Habenaria e diversas outras terrestres como as espécies de Cleistes, Platythelys paranaensis, Sarcoglottis simplex, Cyrtopodium brandonianum e C. pallidum, entre outras. Esse período também marca o maior número de espécies floridas no interior da mata. Florescem em profusão Epidendrum nocturnum, E. difforme, Galleotia ciliata, Pleurothallis ramosa e Cattleya bicolor. Um pouco mais adiante, do meio para o final do período chuvoso florescem Rodriguezia decora, Comparettia coccinea, Epistephium lucidum e Habenaria glaucophylla var. brevifolia. Ao longo deste período o número e a quantidade de terrestres floridas no campo vai decrescendo progressivamente, sendo encontrados em floração Cyrtopodium paludicolum e uma ou outra espécie de Habenaria de floração mais tardia, como $H$. ayangannensis e $H$. mitomorpha.

Outro fator que apresenta forte influência na floração de plantas campestres é a ocorrência de fogo. Espécies como Pelexia goyazensis, Sarcoglottis sagitatta, Platythelys paranaensis, Sacoila lanceolata aff. var. paludicola e Cyanaeorchis minor só foram observadas floridas em áreas anteriormente queimadas. No caso de várias outras como Habenaria leucosantha, Galeandra paraguayensis, Sarcoglottis uliginosa e Cyrtopodium pallidum grandes conjuntos em floração foram sempre observados em áreas anteriormente queimadas, embora um ou outro exemplar também possa ser encontrado em floração em locais não queimados.

O tempo de resposta dessas espécies à passagem do fogo é variável. Alguns táxons respondem pronta e positivamente ao fogo, estando entre as primeiras plantas a florescer após uma queimada, como Pelexia goyazensis, Sarcoglottis sagitatta e Sacoila lanceolata aff. var. paludicola, que florescem até duas semanas após a ocorrência do fogo. Outras florescem após um período maior, como Cyanaeorchis minor, Galeandra paraguayensis, Cyrtopodium fowliei e $C$. pallidum. Outras ainda, como algumas espécies de Habenaria tardias, como $H$. ayangannensis e $H$. mitomorpha, florescem vários meses após a passagem do fogo, mas ainda claramente sob algum efeito secundário deste, uma vez que a floração é sempre mais expressiva em áreas queimadas do que naquelas não queimadas.

Por outro lado, em alguns casos o fogo parece ser claramente prejudicial. É o caso de espécies que florescem no auge do período seco, como Habenaria nuda var. pygmaea e Cyrtopodium parviflorum, época que coincide com as queimadas. Quando a ocorrência do fogo coincide com o período de floração ou frutificação dessas espécies, as inflorescências ou os frutos geralmente são destruídos. Mas, em todo caso, o efeito é fundamentalmente dependente da época exata de queimada. Quando o fogo é precoce, e anterior à floração destas espécies, ocorrendo em junho e julho, pode favorecer a floração. Em suma, o fogo parece ser mais vezes um fator indutor da floração de diversas Orchidaceae campestres do que um fator prejudicial. Entretanto, os mecanismos exatos envolvidos e o seu efeito a longo prazo, tanto sobre os indivíduos como sobre a dinâmica de suas populações, são pouco conhecidos.

Comparação das Orchidaceae da Reserva do Guará com as de outras localidades - Comparada a outras unidades de conservação do Distrito Federal, a REG tem importância secundária, por sua área reduzida. $\mathrm{O}$ Parque Nacional de Brasília (PNB), por exemplo, possui 30.000 ha, a Estação Ecológica de Águas Emendadas 10.000 ha, enquanto o Jardim Botânico de Brasília (JBB) possui 4.429 ha. Todavia, apesar da Reserva corresponder a somente $0,4 \%$ da área total sob conservação efetiva no Distrito Federal, que é de cerca de 50.000 ha (UNESCO 2000), ela abriga $41 \%$ do total de 254 táxons de Orchidaceae registrados para o DF (Batista \& Bianchetti 2003).

Quando comparada com inventários florísticos de outras localidades do DF, a REG se destaca pelo grande número de táxons de Orchidaceae (Tab. 3). Entretanto, esses resultados não passaram por tratamento estatístico específico, principalmente no que se refere à comparação entre unidades amostrais. Eles apontam apenas uma grande concentração de espécies da família Orchidaceae em uma área relativamente pequena, o que não é comum. De qualquer modo, até onde se conhece, não há nenhum outro local no DF, dentro ou fora das unidades de conservação, de tamanho semelhante à Reserva que abrigue número tão expressivo de táxons de Orchidaceae.

O número de espécies de Orchidaceae na R.E. do Guará pode ser explicada pelo fato dela conter, em 
Tabela 3. Número de espécies de Orchidacae registrados para diversas localidades no Brasil.

\begin{tabular}{|c|c|c|c|}
\hline Localidade (Estado) & № gêneros/táxons & Área & Referência \\
\hline Distrito Federal & $72 / 254$ & 578.300 ha & Batista \& Bianchetti 2003 \\
\hline Reserva Ecológica do Guaráa & $44 / 105$ & 147 ha & Este trabalho \\
\hline Parque Nacional de Brasília & $46 / 75^{\mathrm{b}}$ & 30.000 ha & Proença et al. 2001 \\
\hline Reserva Ecológica do IBGE & $36 / 68$ & 1.360 ha & Pereira et al. 1993 \\
\hline Fazenda Sucupira $^{\mathrm{a}}$ & $30 / 68^{b}$ & $1.763 \mathrm{ha}$ & Walter \& Sampaio 1998 \\
\hline ARIE do Riacho Fundo ${ }^{a}$ & $22 / 68^{b}$ & 480 ha & Proença et al. 2001 \\
\hline Fazenda Água Limpa & $18 / 49^{b}$ & $2.660 \mathrm{ha}$ & Proença et al. 2001 \\
\hline Jardim Botânico de Brasília & $23 / 43^{\mathrm{b}}$ & 4.429 ha & Proença et al. 2001 \\
\hline Parque Ecológico Norte ${ }^{a}$ & $9 / 19^{\mathrm{b}}$ & 176 ha & Proença et al. 2001 \\
\hline Estação Ecológica de Águas Emendadas & $13 / 17^{\mathrm{b}}$ & 10.000 ha & Maury et al. 1994 \\
\hline \multicolumn{4}{|l|}{ Cerrado/Campo rupestre extra DF } \\
\hline Lagoa Santa (MG) & $41 / 120$ & ca. $10.800 \mathrm{ha}^{\mathrm{c}}$ & Warming 1892 \\
\hline Serra de São José (MG) ${ }^{\mathrm{a}}$ & $40 / 86$ & ca. 2.500 ha & Alves 1991 \\
\hline PN da Serra do Cipó (MG) & $33 / 80$ & 33.800 ha & Barros 1987 \\
\hline Catolés (BA) & $31 / 76$ & 66.700 ha & Zappi et al. 2003 \\
\hline PN do Caparaó (MG) & $31 / 68$ & 31.853 ha & Leoni 1997 \\
\hline PN da Chapada dos Veadeiros (GO) & $18 / 47$ & 65.000 ha & Munhoz \& Proença 1998 \\
\hline Pico das Almas (BA) & $19 / 46$ & 17.000 ha & Toscano-de-Brito 1995 \\
\hline Parque Estadual do Itacolomi $(\mathrm{MG})^{\mathrm{a}}$ & $24 / 41$ & $7.543 \mathrm{ha}$ & Alves 1990 \\
\hline Mucugê (BA) & $13 / 26$ & 89.600 ha & Harley \& Simmons 1986 \\
\hline RPPN Linda Serra dos Topázios (GO) & $7 / 14$ & 496 ha & Proença et al. 2000 \\
\hline Estação Ecológica do Tripuí (MG) & $7 / 10$ & 337 ha & Pedralli et al. 1997 \\
\hline \multicolumn{4}{|l|}{ Mata Atlântica } \\
\hline Macaé de Cima $(\mathrm{RJ})^{\mathrm{a}}$ & $66 / 270$ & 15.000 ha & Miller et al. 1994 \\
\hline PE das Fontes do Ipiranga (SP) & $52 / 125$ & 345 ha & Barros 1983 \\
\hline Ilha do Cardoso (SP) & $53 / 118$ & 22.500 ha & Barros 1991 \\
\hline \multicolumn{4}{|l|}{ Amazônia } \\
\hline Serra dos Carajás $(\mathrm{PA})^{\mathrm{a}}$ & $55 / 118$ & 429.000 ha & Silveira et al. 1995 \\
\hline Reserva Florestal Adolpho Ducke (AM) & $40 / 78$ & 10.072 ha & Ribeiro et al. 1999 \\
\hline Serra das Andorinhas $(\mathrm{PA})^{\mathrm{a}}$ & $37 / 76$ & 60.000 ha & Atzingen et al. 1996 \\
\hline Reserva Biológica de Campina (AM) ${ }^{\mathrm{a}}$ & $17 / 31$ & 900 ha & Braga 1977 \\
\hline
\end{tabular}

a - Trabalhos específicos sobre Orchidaceae ou locais com esforço especifico de coleta para a família. b - Dados atualizados a partir do exame de exsicatas e de coletas dos autores. c - Segundo Warming (1892) a área explorada em Lagoa Santa foi de 3 leguas ${ }^{2}$ (= 10.800 ha).

área pequena, extensões consideráveis de diferentes fisionomias, tanto campestres quanto florestais, todas favoráveis ao desenvolvimento de orquídeas. Dois exemplos ilustram essa constatação. Um local também rico em espécies de Orchidaceae no DF, é a mata do Acampamento, no Parque Nacional de Brasília, que apresenta número de espécies de mata semelhante ao da REG, devido a diversidade de tipos florestais, embora os campos adjacentes sejam mais uniformes e menos diversos. Por outro lado, a ARIE do Riacho Fundo apresenta quase tantas espécies de Orchidaceae campestres quanto a REG, devido a grande diversidade de tipos campestres, mas as matas são menos diversas e apresentam proporção de espécies menor.

Ao se comparar apenas os dados de inventários de Orchidaceae para localidades fora do DF e mesmo fora do bioma Cerrado (Tab. 3), é revelado que a REG apresenta número de espécies comparável ou superior a alguns de Mata Atlântica, o bioma brasileiro mais rico em espécies de Orchidaceae (Pabst \& Dungs 1975; 1977). Essas comparações são relativas e qualitativas, uma vez que dentro de cada área comparada a distribuição das espécies não é uniforme e as áreas encontram-se diferencialmente amostradas (esforços diferentes de coleta). No entanto, estes dados ressaltam a grande concentração de táxons pertencentes à família na REG e evidenciam que no Cerrado existem localidades com número de espécies comparável a inventários de outros biomas, sabidamente mais propícios ao estabelecimento de exemplares da família.

Conservação e ameaças - Embora constitua uma unidade de conservação, a integridade da Reserva do Guará ainda é seriamente ameaçada por uma série de fatores, dos quais o principal é a pressão humana 
agravada tanto por sua localização como pelo tamanho reduzido da área. O principal problema enfrentado hoje é a ocupação da Reserva como local de moradia por famílias de baixa renda. Decorre daí principalmente a destruição de áreas de campo úmido para implantação de lavouras de subsistência e a retirada de madeira no interior da mata. Outro problema é a coleta de espécies ornamentais. Para algumas espécies mais visadas por colecionadores, como Phragmipedium vittatum e Cattleya bicolor, a coleta predatória tem contribuído significativamente para a redução de suas populações. Outro fator é a dinâmica natural da vegetação, principalmente a retração de áreas de campo limpo inundável e campo limpo estacionalmente úmido e sua substituição por capoeiras. $\mathrm{O}$ resultado é que as Orchidaceae que normalmente ocorrem no campo inundável tendem a desaparecer, uma vez que a capoeira que substitui o campo abriga poucas espécies desta família. Para algumas espécies já raras, como Phragmipedium vittatum e Cyanaeorchis minor observamos o completo desaparecimento de algumas populações. No entanto, apesar da descaracterização progressiva da Reserva, 88 táxons ( $84 \%$ ) foram coletados ou observados no local nos últimos quatorze anos e ainda devem existir em maior ou menor número na área.

\section{Agradecimentos}

A Zenilton J.G. Miranda, Rafael S. Oliveira, Anajulia H. Salles, Maria Aparecida da Silva e Dionisio Alvarenga que contribuíram para o levantamento das Orchidaceae da Reserva Ecológica do Guará com novos registros para o local; a Anajulia H. Salles, pela disponibilização do caderno de coletas de E.P. Heringer; a Bruno M.T. Walter, pela leitura critica, sugestões e correções no artigo; aos curadores dos herbários CEN, HB, HEPH, IBGE e UB, pelos empréstimos ou acesso às suas coleções; a dois revisores anônimos, que apresentaram correções e sugestões que contribuiram para o aprimoramento do trabalho.

\section{Referências bibliográficas}

Alves, R.J.V. 1990. The Orchidaceae of Itacolomi state park in Minas Gerais, Brazil. Acta Botanica Brasilica 4(2): 65-72.

Alves, R.J.V. 1991. Field guide to the orchids of the Serra de São José, Minas Gerais, Brazil. Praga, Tropicaleaf.

Atzingen, N.V.; Cardoso, A.L.R. \& Ilkiu-Borges, A.L. 1996. Flora orquidológica da Serra das Andorinhas, São Geraldo do Araguaia - PA. Boletim do Museu Paraense Emílio Goeldi, série Botânica 12(1): 59-74.
Barros, F. 1983. Flora fanerogâmica da reserva do Parque Estadual das Fontes do Ipiranga (São Paulo, Brasil). 198 - Orchidaceae. Hoehnea 10: 74-124.

Barros, F. 1987. Orchidaceae. Pp. 125-130. In: A.M. Giulietti; N.L. Menezes; J.R. Pirani; M. Meguro \& M.G.L. Wanderley (eds.). Flora da Serra do Cipó, Minas Gerais: caracterização e lista das espécies. Boletim de Botânica da Universidade São Paulo 9(1).

Barros, F. 1991. Orchidaceae. Pp. 142-152. In: M.M.R.F. Melo; F. Barros; M.G.L. Wanderley; M. Kirizawa; S.L. Jung-Mendaçolli \& S.A.C. Chiea (eds.). Flora fanerogâmica da Ilha do Cardoso: Caracterização geral da vegetação e listagem das espécies ocorrentes. v. I. São Paulo, Instituto de Botânica.

Batista, J.A.N. \& Bianchetti, L.B. 2003. Lista atualizada das Orchidaceae do Distrito Federal, Brasil. Acta Botanica Brasilica 17(2): 183-201.

Braga, P.I.S. 1977. Aspectos biológicos das Orchidaceae de uma campina da Amazônia Central. Acta Amazonica (suplemento) 7(2): 1-89.

Castro-Neto, V.P. \& Campacci, M.A. 2000. Icones Orchidacearum Brasilienses I. 100 tab. Coordenadoria das Associações Orquidófilas do Brasil.

Christenson, E.A. 1988. Nomenclatural changes in neotropical orchidaceae. Lindleyana 3(4): 221-223.

Christenson, E.A. 1996. Notes on neotropical orchidaceae II. Lindleyana 11(1): 12-26.

Codeplan. 1984. Atlas do Distrito Federal II. Brasília, Governo do Distrito Federal.

Cogniaux, A. 1893-1896. Orchidaceae. Pp. 1-672. In: C.F.P. Martius; A.G. Eichler \& I. Urban (eds.). Flora Brasiliensis 3(4). Munique, F. Fleischer.

Cogniaux, A. 1898-1902. Orchidaceae. Pp. 1-664. In: C.F.P. Martius; A.G. Eichler \& I. Urban (eds.). Flora Brasiliensis 3(5). Munique, R. Oldenbourg.

Cogniaux, A. 1904-1906. Orchidaceae. Pp. 1-604. In: C.F.P. Martius; A.G. Eichler \& I. Urban (eds.). Flora Brasiliensis 3(6). Munique, R. Oldenbourg.

Dressler, R.L. 1982. The Orchids: natural history and classification. Cambridge, Harvard University Press.

Dressler, R.L. 1993. Phylogeny and classification of the orchid family. Portland, Dioscorides Press.

Garay, L.A. 1977. Systematics of the Physurinae (Orchidaceae) in the new world. Bradea 2(28): 191-204.

Garay, L.A. 1980. A generic revision of the Spiranthinae. Botanical Museum Leaflets Harvard University 28(4): 277-425.

Hágsater, E. 1993. Epidendrum anceps or Epidendrum secundum? Orquídea 13(1-2): 153-158.

Harley, R.M. \& Simmons, N.A. 1986. Florula of Mucugê. Kew, Royal Botanic Gardens.

Hoehne, F.C. 1940. Orchidaceas. Pp. 1-254. In: F.C. Hoehne (ed.). Flora Brasilica 12(1). São Paulo, Secretaria da Agricultura, Indústria e Comércio de São Paulo.

Hoehne, F.C. 1942. Orchidaceas. Pp. 1-218. In: F.C. Hoehne (ed.). Flora Brasilica 12(6). São Paulo, Secretaria da Agricultura, Indústria e Comércio de São Paulo.

Hoehne, F.C. 1945. Orchidaceas. Pp. 1-389. In: F.C. Hoehne (ed.). Flora Brasilica 12(2). São Paulo, Secretaria da Agricultura, Indústria e Comércio de São Paulo. 
Hoehne, F. C. 1953. Orchidaceas. Pp. 1-397. In: F.C. Hoehne (ed.). Flora Brasilica 12(7). São Paulo, Secretaria da Agricultura, Indústria e Comércio de São Paulo.

Leoni, L.S. 1997. Catálogo preliminar das fanerógamas ocorrentes no Parque Nacional do Caparaó, Minas Gerais. Pabstia 8(2): 1-28.

Maury, C.M.; Ramos, A.E. \& Oliveira, P.E. 1994. Levantamento florístico da estação ecológica de Águas Emendadas. Boletim do Herbário Ezechias Paulo Heringer 1: 46-67.

Mendonça, R.C.; Felfili, J.M.; Walter, B.M.T.; Silva Junior, M.C.; Rezende, A.; Filgueiras, T.S. \& Nogueira, P.E. 1998. Flora vascular do cerrado. Pp. 289-556. In: S.M. Sano \& S.P. Almeida (eds.). Cerrado: ambiente e flora. Planaltina, EMBRAPA-CPAC.

Menezes, L.C. 1995a. Novas orquídeas brasileiras. Boletim CAOB 6: 8-13.

Menezes, L.C. 1995b. In memory of Dr. J.A. Fowlie: Cyrtopodium fowliei. Orchid Digest 59: 17-18.

Miller, D.; Warren, R. \& Miller, I.M. 1994. Orchids of the high mountain Atlantic rain forest in southeastern Brazil. Rio de Janeiro, Salamandra.

Munhoz, C.B.R. \& Proença, C.E.B. 1998. Composição florística do município de Alto Paraíso de Goiás na Chapada dos Veadeiros. Boletim do Herbário Ezechias Paulo Heringer 3: 102-150.

Nogueira, P.E.; Nóbrega, M.G.G. \& Pereira da Silva, G. 2002. Levantamento florístico e fisionomias do Parque Ecológico Ezechias Heringer (Parque do Guará) Distrito Federal, Brasil. Boletim do Herbário Ezechias Paulo Heringer 10: 31-56.

Pabst, G.F.J. 1967. Additamenta ad Orchideologiam Brasiliensem - VIII. Orquídea (Rio de Janeiro) 29(1): 5-13.

Pabst, G.F.J. 1976. Additamenta ad Orchideologiam Brasiliensem - XXII. Bradea 2(14): 79-90.

Pabst, G.F.J. \& Dungs, F. 1975. Orchidaceae Brasilienses, v.1. Hildesheim, Brücke-Verlag Kurt Schmersow.

Pabst, G.F.J. \& Dungs, F. 1977. Orchidaceae Brasilienses, v.2. Hildesheim, Brücke-Verlag Kurt Schmersow.

Pedralli, G.; Freitas, V.L.O.; Meyer, S.T.; Teixeira, M.C.B. \& Gonçalves, A.P.S. 1997. Levantamento florístico na estação ecológica do Tripuí, Ouro Preto, MG. Acta Botanica Brasilica 11(2): 191-213.

Pereira, B.A.S.; Aparecida da Silva, M. \& Cunha de Mendonça, R. 1993. Orchidaceae. Pp. 35-36. In: Reserva Ecológica do IBGE, Brasília (DF): lista das plantas vasculares. Rio de Janeiro, IBGE.
Proença, C.E.B.; Oliveira, R.S. \& Silva, A.P. 2000. Flores e frutos do cerrado: Guia de campo ilustrado baseado na flórula da reserva particular do patrimônio natural Linda Serra dos Topázios, Cristalina, Goiás, Brasil. Brasília, Ed. Universidade de Brasília.

Proença, C.E.B.; Munhoz, C.B.R.; Jorge, C.L. \& Nóbrega, M.G.G. 2001. Listagem e nível de proteção das espécies de fanerógamas do Distrito Federal, Brasil. Pp. 89-359. In: T.B. Cavalcanti \& A.E. Ramos (eds.). Flora do Distrito Federal. v.I. Brasília, Embrapa Recursos Genéticos e Biotecnologia.

Ribeiro, J.F. \& Walter, B.M.T. 1998. Fitofisionomias do bioma cerrado. Pp. 89-166. In: S.M. Sano \& S.P. Almeida (eds.). Cerrado: ambiente e flora. Planaltina, Embrapa Cerrados.

Ribeiro, J.E.L.S.; Hopkins, M.J.G.; Vicentini, A.; Sothers, C.A.; Costa, M.A.S.; Brito, J.M.; Souza, M.A.D.; Martins, L.H.P.; Lohmann, L.G.; Assunção, P.A.C.L.; Pereira, E.C.; Silva, C.F.; Mesquita, M.R. \& Procópio, L.C. 1999. Flora da Reserva Ducke: Guia de identificação das plantas vasculares de uma floresta de terra firme na Amazônia Central. Manaus, INPA.

Silveira, E.C.; Cardoso, A.L.R.; Ilkiu-Borges, A.L. \& Atzingen, N.V. 1995. Flora orquidológica da Serra dos Carajás, estado do Pará. Boletim do Museu Paraense Emílio Goeldi, série Botânica 11(1): 75-87.

Spix, J.B. \& Martius, C.F.P. 1828. Reise in Brasilien. Munique. Reedição de 1981. Viagem pelo Brasil, 18171820. v.2. São Paulo, Ed. Itatiaia \& Ed. Universidade de São Paulo.

Toscano-de-Brito, A.L.V. 1995. Orchidaceae. Pp. 725-767. In: B.L. Stannard (ed.). Flora of the Pico das Almas: Chapada Diamantina - Bahia, Brazil. Kew, Royal Botanic Gardens. UNESCO. 2000. Vegetação no Distrito Federal: tempo e espaço. Brasília.

Van den Berg, C. \& Chase, M.W. 2001. Nomenclatural notes on Laeliinae-II. Additional combinations and notes. Lindleyana 16(2): 109-112.

Zappi, D.C.; Lucas, E.; Stannard, B.L.; Lughadha, E.N.; Pirani, J.R.; Queiroz, L.P.; Atkins, S.; Hind, D.J.N.; Giulietti, A.M.; Harley, R.M. \& Carvalho, A.M. 2003. Lista das plantas vasculares de Catolés, Chapada Diamantina, Bahia, Brasil. Boletim de Botânica da Universidade São Paulo 21(2): 345-398.

Walter, B.M.T. \& Sampaio, A.B. 1998. A vegetação da Fazenda Sucupira. Brasília, Embrapa Recursos Genéticos e Biotecnologia.

Warming, E. 1892. Lagoa Santa: Et Bidrag til den biologiske Plantegeografi. Kgl. Danske Vidensk. Selsk. Skr., Raekke, naturvidensk. og math. Afd 6(3). Kjøbenhavn, Bianco Lunos Kgl. Hof-Bogtrykkeri. 